\title{
What Is a Medication-Related Problem? A Qualitative Study of Older Adults and Primary Care Clinicians
}

\author{
Francesca M. Nicosia, $P h D^{1,2,3}$, Malena J. Spar, $M P H^{1,2}$, Marilyn Stebbins, PharmD4, \\ Rebecca L. Sudore, $M D^{1,2}$, Christine S. Ritchie, $M D, M S P H^{1,5}$, \\ Kirby P. Lee, PharmD, MA, MAS4, Kevin Rodondi, PharmD4, and Michael A. Steinman, \\ $M D^{7,2,5}$
}

\begin{abstract}
'Division of Geriatrics, University of California, San Francisco, San Francisco, USA; ${ }^{2}$ San Francisco Veterans Affairs Medical Center, San Francisco, CA, USA; ${ }^{3}$ Institute for Health \& Aging, University of California, San Francisco, San Francisco, USA; ${ }^{4}$ Department of Clinical Pharmacy, School of Pharmacy, University of California, San Francisco, San Francisco, USA; ${ }^{5}$ San Francisco Campus for Jewish Living Center for Research in Aging, San Francisco, USA.
\end{abstract}

\begin{abstract}
BACKGROUND: Older adults often take multiple medications, leading to a myriad of medication-related problems. Addressing these problems requires thoughtful approaches that align with patients' perspectives and experiences.

OBJECTIVE: To (1) identify and categorize medicationrelated problems from the patient perspective and (2) understand patient and clinician attitudes toward these problems and experiences with addressing these problems.
\end{abstract}

DESIGN: Qualitative, semi-structured interviews with patients and focus groups with physicians and pharmacists.

PARTICIPANTS: Twenty older adults recruited from an academic medical center and from a community senior center; 14 primary care physicians and 6 pharmacists affiliated with an academic medical center.

APPROACH: Hybrid deductive-inductive thematic analysis.

KEY RESULTS: Older adults identified a variety of medication-related problems that could be classified into four broad categories: (1) obtaining medications (e.g., problems with cost and insurance coverage); (2) taking medications (e.g., organization and remembering to take pills); (3) medication effects, including side effects and concerns over lack of effectiveness; and (4) communication and care coordination, including information related to medications. Many of the problems described by older adults were framed within the person's socioemotional context, including the impact of medications on interpersonal relationships, emotional wellbeing, and activities that add meaning and quality to life. In contrast, clinicians almost exclusively focused on discrete medication issues without reference to this larger context and expressed relatively little interest in learning more about their patients' perspectives.

CONCLUSIONS: Older adults experience medicationrelated problems as inseparable from their broader life

Electronic supplementary material The online version of this article (https://doi.org/10.1007/s11606-019-05463-z) contains supplementary material, which is available to authorized users.

Received October 31, 2018

Revised May 28, 2019

Accepted September 25, 2019

Published online November 1, 2019 context. Incorporating the social and emotional context of medications and related communication into a problem-focused framework can guide clinicians in specific actions and interventions to address medicationrelated problems from the patient perspective.

KEY WORDS: geriatrics; primary care; qualitative research; medication; medication-related problems.

J Gen Intern Med 35(3):724-31

DOI: $10.1007 / \mathrm{s} 11606-019-05463-\mathrm{Z}$

(c) Society of General Internal Medicine 2019

\section{INTRODUCTION}

Older adults often take multiple medications, leading to a myriad of medication-related problems. ${ }^{1}$ Previous work to address medication-related problems has largely relied on clinician-focused perspectives on these issues. ${ }^{2-4}$ For example, physicians and pharmacists interested in improving pharmaceutical care have traditionally expressed concern about issues such as medication non-adherence, inappropriate prescribing, and adverse reactions, which each affects $5-50 \%$ of older adults and can result in substantial morbidity. ${ }^{5-7}$ Clinicians have then developed interventions to address these issues, with less attention to what types of medication-related problems are most pressing to patients and whether these interventions align with patient perspectives, priorities, and experiences of medication use..$^{8-10}$ This is not to say that patients' concerns have been entirely ignored. For example, efforts to improve medication adherence and reduce adverse drug events include models of shared decision-making and strategies to enhance medication communication and concordance between patients and clinicians. ${ }^{8,11-15}$ However, the core problems that these efforts try to address (e.g., nonadherence) have typically been identified, a priori, based on clinician or payer perspectives. Such clinician-focused perspectives are exemplified by the numerous taxonomies that have been developed to categorize medication-related problems, which almost universally reflect clinician-focused 
perspectives on what constitutes a medication-related problem that is worth a clinician's time and attention. ${ }^{16}$

While clinician priorities are important, the relative lack of input from older patients limits opportunities to improve patient-centered outcomes. ${ }^{17}$ This may happen for several reasons, including suboptimal communication around medications during clinical encounters. ${ }^{18-20}$ Patients may be reluctant to disclose medication-related issues, especially those related to psychosocial factors which are not often solicited by primary care physicians. ${ }^{18,20-23}$ Further, clinician priorities around medications can differ from those considered most important by their older patients.

A growing body of literature on patients' lived experiences with medications highlights the need for clinicians to better understand medication-related problems from the patient perspective. $^{21,23-25}$ In a recent systematic review and metasynthesis of qualitative studies, researchers developed a conceptual model of patients' lived experience of medicines (PLEM), including how medication experience influences patients' health and well-being, beliefs, and behavior. ${ }^{21}$ However, these findings have not been easily translated into practice in primary care settings, where there still exists a substantial divide between the full lived experience of older adults with their medications and the reductionistic, problem-oriented approaches commonly favored by physicians. This gap must be addressed if we are to successfully inform interventions that address the problems and challenges that patients have with their medications.

To help bridge this gap, we conducted a qualitative study among older adults, primary care physicians, and pharmacists so as to better understand patient and clinician perspectives on medication-related problems and approaches to addressing them. Our goals were to (1) identify and categorize medication-related problems from a patient perspective and (2) understand patient and clinician attitudes toward these medication-related problems and their experiences with addressing these problems.

\section{METHODS}

Between November 2016 and August 2017, a medical anthropologist (FMN) conducted 20 semi-structured interviews with older adults to facilitate in-depth understanding of individual experiences with and attitudes toward problems with medications. ${ }^{26}$ We also conducted four focus groups with 20 clinicians (3 focus groups each with 4-6 primary care physicians, 1 with 6 pharmacists) to explore clinicians' perspectives on and attitudes toward the problems they thought were most troublesome for their older patients; focus groups were used for clinicians instead of interviews largely for logistical reasons, although with the expectation that either method would provide valuable information. ${ }^{27}$ Focus groups were facilitated by a medical anthropologist (FMN) and a geriatrician-researcher (MAS) with assistance from a clinical research coordinator trained in qualitative methods
(MJS). The study was approved by the institutional review boards (IRBs) of the University of California, San Francisco and the San Francisco VA Medical Center.

\section{Study Population}

Eligibility criteria for older adults included the following: age $\geq 65$ years, taking at least one medication, having at least one self-reported medication-related problem, and ability to pass a cognitive screen. ${ }^{28}$ First, we recruited patients from primary care clinics affiliated with an academic medical center. Due to IRB requirements, we sent an opt-in letter to a randomly selected sample of eligible patients. Of 700 letters sent, 33 patients contacted study personnel; 10 were eligible based on a screening questionnaire, all of whom enrolled in the study and completed the interview. The most common reason for exclusion $(n=16)$ was the absence of a current medication-related problem. Second, after determining that our initial recruitment method was selecting more highly educated participants, we purposefully selected a community senior center that served a more diverse population of older adults and recruited participants in person. ${ }^{29}$ Ten were eligible based on a screening questionnaire, all of whom enrolled in the study and completed the interview. The most common reasons for exclusion $(n=$ 8) were the absence of a current medication-related problem and lack of English proficiency.

Eligibility criteria for clinicians included caring for older adults in an ambulatory clinical practice. We used emails to recruit all eligible primary care physicians from an academic medical center-based general internal medicine practice and from a community-based, academic family medicine practice. To recruit clinical pharmacists affiliated with this academic medical center, a clinical pharmacist and study co-author (MRS) identified pharmacists across multiple clinics whose clinical practice included ambulatory older adults. In this paper, we use the term "clinicians" to refer to physicians and pharmacists collectively, and the individual professional identifier (e.g., "pharmacists") when referring specifically to one group.

\section{Data Collection}

Interviews with older adults focused on (1) eliciting problems and concerns related to medications and (2) their efforts to address their problems either with their primary care providers or on their own (see Online Appendix 1). We approached interviews without an a priori definition of what constitutes a medication-related problem and first asked open-ended questions about the most important and/or bothersome concerns related to any aspect of their medications experienced by older adults. Follow-up questions were informed by known medication-related problems from the literature and clinical experience..$^{21,23,24,30-32}$ Interviews averaged $60 \mathrm{~min}$ and took place either in participants' homes or a private room in a clinical research center.

Three focus groups with 4-6 physicians and one focus group with 6 pharmacists lasted 60-90 min and elicited perspectives on types of medication-related problems experienced by their older 
patients and specific strategies, tools, and barriers to address these problems (see Online Appendix 2). Interviews and focus group discussions were audio recorded and professionally transcribed, and transcriptions were imported into Atlas.ti (Scientific Software, Berlin, Germany, version.7.5.18). All participants received a gift card for their participation.

\section{Approach}

We analyzed transcripts iteratively using a hybrid deductive and inductive approach to qualitative thematic analysis. ${ }^{33}$ This systematic approach to analyzing qualitative data allowed us to categorize medication problems and to identify emergent constructs, patterns, and themes derived from participants' own words. First, we developed an initial codebook based on the study goals and commonly known types of medication-related problems. FN, MJS, and MAS openly coded three transcripts of patient interviews using three broad code categories: (1) patient-identified medication-related problems, (2) attitudes toward these problems, and (3) actions taken to address these problems. During the coding process, inductive codes were assigned to segments of the transcripts that described new concepts. This was followed by parallel independent coding by FN and MJS of five additional transcripts to determine the applicability of the initial coding scheme. Thereafter, three coders independently reviewed each transcript (FN, MJS, and MAS). Through regular team meetings, we refined the codebook by reviewing the accuracy of code definitions and their appropriate application, recoding segments as necessary, and iteratively developing themes. We recruited subjects until thematic saturation was reached, consistent with the goal of qualitative research to provide thematic evaluation rather than broad generalizability. We analyzed focus group transcripts using a similar process and paid close attention to new codes and themes not identified in patient interviews. Using these findings, we iteratively organized medication-related problems into categories and identified themes within each category. To ensure validity, dependability and establish inter-coder agreement, we met weekly and resolved disagreements in coding and interpretation through discussion and consensus. We discussed interpretations of findings with the full study team throughout the analysis process. ${ }^{34}$

\section{RESULTS}

\section{Participant Characteristics}

Among 20 older adults interviewed, the mean age was 80 years $(\mathrm{SD} \pm 7), 80 \%$ took 6 or more medications, and $95 \%$ had some college or higher education level (Table 1). Among 14 primary care physicians and 6 pharmacists, $43 \%$ of physicians had been in practice more than 10 years and $50 \%$ of pharmacists had been in practice for over 20 years (Table 2).
Table 1 Patient Characteristics $(n=20)$

\begin{tabular}{ll}
\hline \hline Characteristics & Patients $(\mathbf{n}=\mathbf{2 0})$ \\
\hline Age, mean years (standard deviation) & $80( \pm 7)$ \\
Female, $n(\%)$ & $12(60 \%)$ \\
Race/ethnicity, $n(\%)$ & $15(75 \%)$ \\
White & $1(5 \%)$ \\
Black, AA & $1(5 \%)$ \\
Latino/Hispanic & $3(15 \%)$ \\
Asian/Pacific Islander & \\
Level of education, $n$ (\%) & $1(5 \%)$ \\
HS diploma, GED or less & $6(30 \%)$ \\
Some college & $7(35 \%)$ \\
College graduate & $6(30 \%)$ \\
Graduate degree & \\
Chronic medical conditions (self-reported), $n(\%)$ & $14(70 \%)$ \\
High blood pressure or hypertension & $1(5 \%)$ \\
Diabetes or high blood sugar & $5(25 \%)$ \\
Cancer, not including minor skin cancers & $4(20 \%)$ \\
Chronic lung disease & $5(25 \%)$ \\
Heart problems & $6(30 \%)$ \\
Abnormal heart rhythm & $2(10 \%)$ \\
Stroke & $3(15 \%)$ \\
Emotional, nervous, or psychiatric problems & $16(80 \%)$ \\
Arthritis or rheumatism & $9(45 \%)$ \\
Osteoporosis or fragile bones & $3(15 \%)$ \\
Number of medications (self-reported), $n(\%)$ & $1(5 \%)$ \\
1-2 & $9(45 \%)$ \\
3-5 & $7(35 \%)$ \\
6-9 $10-15$ & $7(35 \%)$ \\
Hospitalized in the last year, $n$ (\%) & $10(50 \%)$ \\
Recruitment method, $n(\%)$ & $10(50 \%)$ \\
Academic primary care clinic (direct mail opt-in) \\
Senior center (tabling event) & \\
\hline
\end{tabular}

\section{Medication-Related Problems Experienced by Older Adults}

Medication-related problems described by older adults and clinicians could be grouped into 4 broad categories: (1) obtaining medications (e.g., problems with cost and insurance coverage); (2) taking medications (e.g., organization and remembering to take pills); (3) medication effects, including side effects and concerns about effectiveness; and (4) communication and care coordination related to medications, including

Table 2 Provider Characteristics $(n=20)$

\begin{tabular}{lll}
\hline \hline Characteristics & $\begin{array}{l}\text { MDs (3 focus } \\
\text { groups; } \boldsymbol{n}=\mathbf{1 4})\end{array}$ & $\begin{array}{l}\text { PharmDs (1 focus } \\
\text { group; } \boldsymbol{n}=\mathbf{6})\end{array}$ \\
\hline Female, $n(\%)$ & $6(43 \%)$ & $6(100 \%)$ \\
Years in practice, $n(\%)$ & $8(57 \%)$ & $2(33 \%)$ \\
$0-9$ & $1(7 \%)$ & $1(17 \%)$ \\
$10-19$ & $5(36 \%)$ & $3(50 \%)$ \\
$>20$ & $10(71 \%)$ & \\
Specialty, $n(\%)$ & & \\
General internal & $4(29 \%)$ & $5(83 \%)$ \\
medicine & & $1(17 \%)$ \\
Family medicine & & \\
General clinical & & $2(33 \%)$ \\
pharmacy & & $2(33 \%)$ \\
Other & $1(7 \%)$ & $0(0 \%)$ \\
Percent of work involving direct patient care, & $(\%)$ \\
$01-20$ & $6(43 \%)$ & $1(17 \%)$ \\
$21-40$ & $3(21 \%)$ & $1(17 \%)$ \\
$41-60$ & $3(21 \%)$ & \\
$61-80$ & $1(7 \%)$ & \\
$81-100$ & &
\end{tabular}


insufficient information about medications. Table 3 shows these four categories and representative types of problems and themes within each area. Within each category, we describe themes that arose from patient and clinician perspectives on these medication-related problems.

\section{Problem Category \# 1: Obtaining Medications}

The first category of problems related to obtaining medications, including challenges paying for medications, managing prior authorization processes, and navigating obtaining refills and renewals.

Financial Barriers and Their Socioemotional Impact. Cost and insufficient insurance coverage were common issues related to older adults' problems with obtaining medications. A key theme was the social and emotional impact associated with the cost of medications. For example, because of the high cost of medication, some participants expressed fear and anxiety around not being able to afford rent or groceries. One 73-year-old woman explained how her medications, "got too expensive, but I got to buy them. That's why I start[ed] going to the [food] pantry. Eventually, I'll probably have to sell [my house]. And I don't want to move, start with new friends and new ways to live. I'm

Table 3 Categories of Medication-Related Problems, with Representative Problems and Themes Within Each Category

\footnotetext{
Problems with obtaining medications

Problems with medication costs and/or insurance coverage

Expired medications/disposal issues

Problems related to prior authorization for medications

Problems getting to the pharmacy

Problems understanding and obtaining refills and renewals

Theme: financial barriers and their socioemotional impact

Theme: refills, renewals, and prior authorizations

Problems with taking medications

Forgetting to take medications

Problems with organizing medications

Problems with medication dosing/frequency/schedule

Problems administering medications (e.g., large pills difficult to

swallow)

Theme: forgetting to take medications: adherence or existential issue?

Problems with medication effects

Concerns about whether medications are effective

Problems with current side effects of medications

Concerns that long-term medication use may induce future side effects

Concerns about interactions between medications

Theme: the inseparability of side effects from patients' overall health,

identity, and daily life

Theme: concern about future side effects

Theme: worries about medication effectiveness

Problems with communication, care coordination, and medication information

Communication between patients and clinicians

Poor relationship with primary care provider leads to uncertainty, concerns, or distrust of medications

Lack of an advocate during appointments leads to suboptimal communication and decisions around medications

Conflicts between patients and clinicians over whether to stop a medication

Confusing medication information (e.g., prescription inserts, advertisements)

Communication challenges within the healthcare system

Difficulties communicating among multiple prescribers leads to suboptimal medication use

Theme: poor communication impacts patients' experience of medication-related problems

Theme: medication-related problems arising from communication challenges within healthcare systems
}

too old for that." Although clinicians identified cost as a barrier to obtaining medications, they did not elaborate on the ripple effects of financial burdens within the broader context of their patients' lives.

Refills, Renewals, and Prior Authorizations. Clinicians commonly raised as a medication-related problem their patients' difficulty obtaining refills or renewals. Clinicians discussed how older adults often had difficulty getting to the pharmacy and experienced confusion over when and how to obtain refills or renew prescriptions. Clinicians also discussed their own challenges and frustrations with obtaining and managing required prior authorizations from insurance companies before prescribing or renewing certain medications. In contrast, challenges with refills, renewals, and prior authorization were not a major theme discussed by patients.

\section{Problem Category \#2: Taking Medications}

The second category of medication-related problems addressed challenges taking medication, including issues such as remembering when and how to take medications.

Forgetting to Take Medications: Adherence or Existential Issue?. Forgetting to take medications was a common problem identified by both patients and clinicians. Clinicians tended to describe patients' memory problems matter-offactly. For example, one physician summarized this sentiment: "[O]ne of the biggest [problems] is memory. Either remembering to take their medicine or remembering if they have already taken them or not." Clinicians commonly framed memory issues as problems of adherence.

In contrast, older adults seldom framed forgetting to take medications as non-adherence. Patients often described forgetting to take medications in affective and existential terms, including worry, being angry with oneself, or that forgetting foreshadowed cognitive decline. One 77-year-old man explained how forgetting medications affected him emotionally: "It's upsetting about what it tells me about my memory. I don't like it at all." Patients described numerous strategies to remember to take medications, including using pill organizers and creative reminders or "tricks" to stay on top of complicated dosing regimens. Despite these strategies, many patients continued to encounter confusion. As one patient said, "Every time I think I have it figured out, I forget something."

When asked to describe their degree of concern over forgetting medications, patients commonly distinguished between "critical" and "less-important" medications. Some explained how they did not consider skipping preventative medications (e.g., hypertension and cholesterol medications) as problematic, citing an absence of adverse effects or symptoms experienced due to missed doses.

\section{Problem Category \#3: Medication Effects}

The third category refers to problems regarding the beneficial and adverse effects of medications, including concerns that 
medications were not helpful or not needed, current adverse effects, and fear of potential future adverse effects.

The Inseparability of Side Effects from Patients' Overall Health, Identity, and Daily Life. Side effects (i.e., adverse drug effects) were the most common medication-related problem discussed by both older adults and clinicians. While clinicians discussed the occurrence of side effects in matterof-fact ways, older adults overwhelmingly discussed side effects within the broader context of their overall health and wellbeing. In describing challenges with her diuretic medications, an 82-year-old woman explained, "If I'm going to [drive an hour away], I have to stop on the way, get off the road and go to [the toilet] somewhere. So I either try to hold it [or] I have to not take the water pill. [My] granddaughter lives an hour away. I ain't been to see her but much this year." Other patients discussed taking medications differently than prescribed as an active strategy to mitigate or reduce unwanted side effects.

A related theme among patients was how the effects of medication use impacted their identity. For example, a 77year-old man who was an avid sailor and cyclist explained how in recent years his medical conditions combined with medication side effects prevented him from riding his bicycle, negatively affecting his sense of self: "My doctor would prefer I didn't ride a bicycle [because of medications] but if I don't ride a bicycle, I just feel useless."

Concern About Future Side Effects. In addition to current side effects, patients frequently expressed concerns over the potential for future, long-term side effects (e.g., liver damage caused by statins). For example, one 77-year-old woman noted, "You can't stay on [the medication] too long because then something else might happen. I don't need any more problems." In contrast, no clinicians mentioned patients' concerns with potential future side-effects.

Worries About Medication Effectiveness. Concerns about effectiveness of medications were another common theme among older adults. Some patients judged medication effectiveness based on the persistence of symptoms related to the targeted condition. Others explained how they would be "happy to pay a little bit more" or tolerate side effects in exchange for effective medications. In contrast, clinicians took medication effectiveness for granted and did not identify it as a medication-related problem that patients might experience. Older adults also described negative psychological and emotional effects from medication therapy they perceived as ineffective. For example, one woman described feelings of frustration and despair after years of unsuccessfully attempting to treat urinary incontinence. She explained, "what makes it so bad is that by these drugs not really being effective, I don't get a decent night's sleep which is just very bad. I used to wake up bounding out of bed glad that I was here to enjoy hopefully another day of being alive, but this has really been a problem."

\section{Problem Category \#4: Communication, Care Coordination, and Information About Medications}

The final problem category covers problems related to communication, coordination, and information around medications.

Poor Communication Impacts Patients' Experience of Medication-Related Problems. Older adults described many of their medication-related problems through the lens of the patient-provider relationship and physicians' communication style. For example, several patients described the importance of trust and rapport with their doctors as a critical element of their medication-related problems. One 86-year-old retired nurse said, "No one tells you anything. 'You take this like a good girl and don't ask me anything, don't ask questions.' It's like a pat on the head. It really infuriates me." Others explained how they had to be "self-reliant" and "stand up" for what they wanted when communicating with their primary care provider about medication problems. Patients also highlighted how an overwhelming amount of information about medications (e.g., pharmacy inserts and advertisements), provoked feelings of confusion and fear that were not adequately addressed by physicians.

Pharmacists echoed patients' concerns related to communication and the patient-provider relationship. Most pharmacists described experiences with patients who were uncomfortable discussing medication problems with their physician or whose physicians did not have adequate time to ask about or address their problems. One pharmacist explained: "Sometimes, [patients will]... divulge things to us that they don't tell their doctor. Like they're not really taking their statin anymore because it's too expensive, or they don't think they need it, but they told their doctor that they are taking it. So there's this trust factor that they have with us that [they can tell] the truth about their medicines." Physicians, on the other hand, did not identify poor communication as a medication-related problem experienced by patients.

Medication-Related Problems Arising from Communication Challenges Within Healthcare Systems. Physicians and pharmacists often noted medication-related problems that arose from suboptimal communication among health care providers. For example, clinicians discussed poor communication among multiple prescribers around medication choices and barriers involving the electronic medical record, including lack of interoperability between health care systems and community pharmacies. In contrast, few older adults mentioned concerns about pharmacy and health care system communication; those who did focused on poor communication and coordination between primary care and specialists.

\section{Cross-cutting Themes}

In addition to the themes related to specific categories and types of medication-related problems, several themes cut across aspects of patients' experience of medication-related problems. 
Divergent Patient and Clinician Perspectives About Medication-Related Problems. Older adults, physicians and pharmacists described medication-related problems in notably different ways. While older adults mentioned a broad array of problems that shaped their lived experience of medications, clinicians focused almost exclusively on issues that fit within a narrow, problem-oriented framework. Moreover, although clinicians were asked to identify and comment on medication-related problems experienced by their older patients, physicians in particular spent a good deal of time discussing their own problems and frustrations - most notably their challenges with medication reconciliation and frustrations communicating with community pharmacies. The overlap and divergence between the problems discussed by patients and physicians are illustrated in Figure 1.

When asked about the extent to which they might be unaware of the kinds of medication-related problems their patients were experiencing, many physicians expressed a lack of curiosity about these "unknown" problems. These physicians noted that the "known" problems already posed major challenges for them and they were unenthusiastic about trying to uncover still more problems that needed to be solved. This was compounded by the lack of time, as another physician said: "Even though the idea of being patient-centered and listening is so important, it's, like, where do you find the time?"

In contrast to physicians, the kinds of medication-related problems discussed by pharmacists were often more closely aligned with the concerns raised by older adults, particularly for communication-related problems. For example, pharmacists spoke at length about how medication-related problems in older adults often arose from challenges in the patientphysician relationship, an issue that was frequently mentioned in interviews with older adults but was not brought up in the physician focus groups.

Medication-Related Issues: Not Always a Problem. Many older adults who described experiencing medication-related issues did not consider these to be problems. For example, some experienced adverse effects from medications but did not frame these effects as problematic or bothersome. Instead, they had a general expectation that medicines have side effects that older people simply need to live with. Others explained how the trust they had in their clinicians mediated the degree to which they felt bothered by issues like side effects (e.g., taking the medicine was good for them because that's what their physician recommended).

\section{DISCUSSION}

Our findings suggest that medication-related problems described by older adults can be grouped into 4 broad categories: (1) obtaining medications; (2) taking medications; (3) medication effects; and (4) communication, care coordination, and information related to medications. Within each of these categories of medication-related problems, older adults often described medication-related problems in ways that were inextricable from the broader, socioemotional context of their lives. This marks a major contrast from existing taxonomies for categorizing medication-related problems, which focus almost exclusively on discrete and highly tangible problems such as non-adherence or adverse drug effects, and lack attention to problems articulated by patients related to fear, communication, and the social and emotional impacts of medication use.

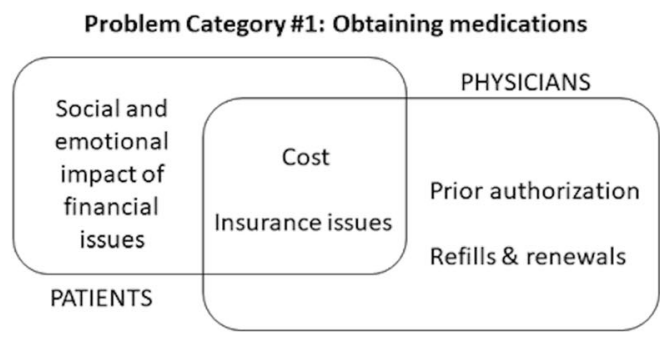

Problem Category \#3: Medication effects

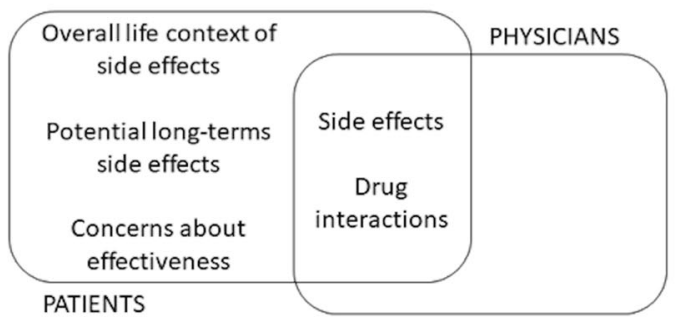

Problem Category \#2: Taking medications

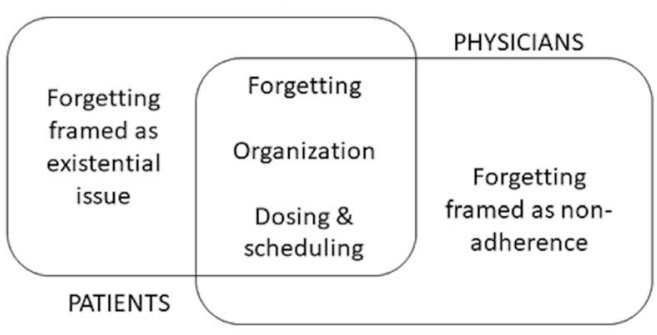

Problem Category \#4: Communication, coordination, and medication information

\begin{tabular}{|c|c|c|}
\hline Poor communication & & PHYSICIANS \\
\hline Poor patient-physician & Lack of time & $\begin{array}{c}\text { Medication } \\
\text { reconciliation }\end{array}$ \\
\hline $\begin{array}{l}\text { Confusing drug } \\
\text { information }\end{array}$ & $\begin{array}{c}\text { Multiple } \\
\text { prescribers }\end{array}$ & $\begin{array}{c}\text { Poor } \\
\text { communication } \\
\text { within health }\end{array}$ \\
\hline PATIENTS & & care system \\
\hline
\end{tabular}

Figure 1 Overlap between older adults' and physicians' perspectives on types of medication-related problems. In each of the four main categories of medication-related problems, the diagrams show commonly raised issues and themes by participant type. Results from pharmacist focus groups are not included in this figure. 
Many medication-related problems described by older adults had two components - the discrete, medication-specific problem itself, and the broader impact of this problem on their lived experience. For example, some problems in each of the four categories were described in concrete terms, such as high cost or adverse effects from a specific medication. However, patients often framed many problems with obtaining or taking medications within the context of their social functioning and emotional well-being, including financial burdens and negative effects on self-image, social functioning, and activities that add meaning and quality to life. Moreover, several older adults also expressed fear around future side effects from long-term medications, and concern over whether their medications were effective. And, while suboptimal communication between patients and clinicians is a well-known problem, ${ }^{35,36}$ our study found that patients framed communication itself as a medication-related problem, not an ancillary concern.

These problems and concerns stand in contrast to the types of medication-related problems identified by physicians. Although some clinicians understood that older adults' medication experience included negative impacts on their daily lives, physicians' recognition of the scope of socioemotional context was limited compared to the breadth of patient-identified problems in this area. This is consistent with previous literature which has identified that psychosocial concerns are seldom solicited by physicians. ${ }^{18}$ In addition, problems such as fear of future side effects and poor communication were seldom discussed by physicians. While many clinicians might not consider these as medication-related problems per se, the fact that patients did suggests that taking a broader view of medication-related problems may be important for meeting the needs and concerns of patients.

To successfully address medication-related problems from a patient-centered approach, interventions must incorporate a more nuanced understanding of these problems to include the broader life context of patients' medication experience in addition to the role that patient-clinician relationships and communication play in creating and resolving these problems. ${ }^{22,31,32,37}$ An essential component of such interventions will likely involve strategies to make clinicians aware of patient concerns and perspectives about their medications, and giving those clinicians skills in responding to them. Physicians may also not be the optimal target, since not only is physician behavior difficult to change, but physicians' focus on their own problems and relative lack of interest in patients' perspectives may detract from their willingness or ability to approach medication-related concerns from a patient-centered perspective. In contrast, there is an opportunity leverage the expertise of other health care professionals such as pharmacists, nurses, and social workers to engage patients around the issues of concern to them, and either address them directly or help patients to navigate such discussions with their physicians. Interprofessional models of integrated primary care are a promising avenue for understanding and addressing patients' medication-related problems, as pharmacists and nurses can be efficient and effective in supporting medication management for older adults. ${ }^{38,39}$

There are several limitations of our study. We experienced low response rates to our initial opt-in patient recruitment letter, and despite our attempts to recruit a diverse population, our sample was majority white and highly educated. The applicability of our findings to more historically disadvantaged populations is unclear; however, other studies have shown that improving communication about medications and trust with providers is important among older patients with lower socioeconomic status. ${ }^{35}$ Further, generalizability is not the goal of qualitative research and we reached thematic saturation for key themes. Because we were interested broadly in types of medication-related problems irrespective of diagnoses, we did not conduct in-depth analysis of specific medications. Physicians in this study were all from universityaffiliated medical practices, potentially limiting the relevance to non-academic settings. Finally, we conducted only one focus group with pharmacists. Their experiences might not reflect those of all pharmacists, and further research is needed to better understand pharmacists' perspectives on addressing medication-related problems in primary care practice.

The widely differing perspectives between older adults and physicians about medication-related problems, including patients' frequent focus on the social and emotional context and communication-driven nature of these problems, suggest that new approaches are needed. By bringing greater awareness to the broader socioemotional context of patients' medication experience, we can begin to bridge the gap between more patient-focused and clinician-derived models of medication problems. To succeed in bridging this gap, these concepts must be incorporated by clinicians, intervention developers, and health systems into a problem-focused framework that can guide specific actions and interventions to assess and address medication-related problems from the patient perspective.

Corresponding Author: Francesca M. Nicosia, PhD; San Francisco Veterans Affairs Medical Center, 4150 Clement St., San Francisco, CA 94121, USA (e-mail: Francesca.Nicosia@ucsf.edu).

Funding Drs. Steinman and Ritchie: Tideswell at UCSF. Dr. Steinman: National Institute on Aging, National Institutes of Health (P3O AG044281, K24AG049057, and R24AG064025). Dr. Sudore is funded in part by the National Institute on Aging, National Institutes of Health (K24AG054415).

\section{Compliance with Ethical Standards:}

The study was approved by the institutional review boards (IRBs) of the University of California, San Francisco and the San Francisco VA Medical Center.

Conflict of Interest: Dr. Steinman served as a consultant for iodine. com, an internet startup company focused on collecting and sharing patient-reported information on medication effectiveness and harms. Dr. Rodondi is an advisor to Arine.io, a medication therapy management company. All remaining authors declare that they do not have a conflict of interest 


\section{REFERENCES}

1. Tran VT, Montori VM, Eton DT, Baruch D, Falissard B, Ravaud P Development and description of measurement properties of an instrument to assess treatment burden among patients with multiple chronic conditions. BMC Med. 2012 Jul 4;10:68.

2. Patterson SM, Cadogan CA, Kerse N, Cardwell CR, Bradley MC, Ryan C, Hughes C. Interventions to improve the appropriate use of polypharmacy for older people. Cochrane Database Syst Rev. 2014 Oct 7:CD008165.

3. Rollason V, Vogt $\mathbf{N}$. Reduction of polypharmacy in the elderly: a systematic review of the role of the pharmacist. Drugs Aging. 2003;20:817-32.

4. Viswanathan M, Kahwati LC, Golin CE, Blalock SJ, Coker-Schwimmer E, Posey R, Lohr KN. Medication therapy management interventions in outpatient settings: a systematic review and meta-analysis. JAMA Intern Med. 2015 Jan;175:76-87.

5. Marcum ZA, Gellad WF. Medication adherence to multidrug regimens. Clin Geriatr Med. 2012;28:287-300.

6. Gurwitz JH, Field TS, Harrold LR, Rothschild J, Debellis K, Seger AC, Cadoret C, Fish LS, Garber L, Kelleher M, Bates DW. Incidence and preventability of adverse drug events among older persons in the ambulatory setting. JAMA. 2003;289:1107-16.

7. Opondo D, Eslami S, Visscher S, de Rooij, Verheij R, Korevaar JC, Abu-Hanna A. Inappropriateness of medication prescriptions to elderly patients in the primary care setting: a systematic review. PLoS One 2012;7:e43617.

8. Fried TR, McGraw S, Agostini JV, Tinetti ME. Views of older persons with multiple morbidities on competing outcomes and clinical decisionmaking. J Am Geriatr Soc. 2008 Oct;56:1839-44.

9. Fried TR, Tinetti ME, Iannone L. Primary care clinicians' experiences with treatment decision making for older persons with multiple conditions. Arch Intern Med. 2011 Jan 10;171:75-80.

10. Willeboordse F, Hugtenburg JG, Schellevis FG, Elders PJ. Patient participation in medication reviews is desirable but not evidence-based: a systematic literature review. Br J Clin Pharmacol. 2014 Dec;78:1201-16.

11. Tinetti ME, McAvay GJ, Fried TR, Allore HG, Salmon JC, Foody JM, Bianco L, Ginter S, Fraenkel L. Health outcome priorities among competing cardiovascular, fall injury, and medication-related symptom outcomes. J Am Geriatr Soc. 2008 Aug;56:1409-16.

12. Patton DE, Cadogan CA, Ryan C, Francis, JJ, Gormley GJ, Passmore $\mathbf{P}$, Kerse N, Hughes CM. Improving adherence to multiple medications in older people in primary care: Selecting intervention components to address patient-reported barriers and facilitators. Health Expect. 2018 Feb;21:138-48.

13. Garavalia L, Garavalia B, Spertus JA, Decker C. Medication Discussion Questions (MedDQ): developing a guide to facilitate patient-clinician communication about heart medications. J Cardiovasc Nurs. 2011;26:E12-9

14. Weingart SN, Gandhi TK, Seger AC, Seger DL, Borus J, Burdick E, Leape LL, Bates DW. Patient-reported medication symptoms in primary care. Arch Intern Med. 2005; 165:234-40.

15. Brown M, Frost R, Ko Y, Woosley R. Diagramming patients' views of root causes of adverse drug events in ambulatory care: an online tool for planning education and research. Patient Educ Couns. 2006;62:302-15

16. Basger BJ, Moles RJ, Chen TF. Development of an aggregated system for classifying causes of drug-related problems. Ann Pharmacother 2015;49:405-18.

17. Britten N, Stevenson FA, Barry CA, Barber N, Bradley CP. Misunderstandings in prescribing decisions in general practice: qualitative study. BMJ. 2000 Feb 19;320:484-8.

18. Joensson ABR, Guassora AD, Freil M, Reventlow S. What the doctor doesn't know: Discarded patient knowledge of older adults with multimorbidity. Chronic Illn. 2018 Sep 13:1-14.
19. Tarn DM, Mattimore TJ, Bell DS, Kravitz RL, Wenger NS. Provider views about responsibility for medication adherence and content of physician-older patient discussions. J Am Geriatr Soc. 2012;60:1019-26.

20. Richard C, Lussier MT. Measuring patient and physician participation in exchanges on medications: Dialogue Ratio, Preponderance of Initiative, and Dialogical Roles. Patient Educ Couns. 2007;65:329-41.

21. Mohammed MA, Moles RJ, Chen TF. Medication-related burden and patients' lived experience with medicine: a systematic review and metasynthesis of qualitative studies. BMJ Open. 2016 Feb 2;6:e010035.

22. Weiner SJ, Schwartz A, Weaver F, Goldberg J, Yudkowsky R, Sharma G, Binns-Calvey A, Preyss B, Schapira MM, Persell, SD, Jacobs E, Abrams RI. Contextual errors and failures in individualizing patient care: a multicenter study. Ann Intern Med. 2010;153:69-75.

23. Shoemaker SJ, Ramalho de Oliveira D. Understanding the meaning of medications for patients: the medication experience. Pharm World Sci. 2008 Jan;30:86-91.

24. Pound P, Britten N, Morgan M, Yardley L, Pope C, Daker-White G, Campbell R. Resisting medicines: a synthesis of qualitative studies of medicine taking. Soc Sci Med. 2005 Jul;61:133-55.

25. Krska J, Morecroft CW, Poole H, Rowe PH. Issues potentially affecting quality of life arising from long-term medicines use: a qualitative study. Int J Clin Pharm. 2013:35:1161-9.

26. Patton MQ. Qualitative research and evaluation methods. Thousand Oaks, CA: SAGE; 2002

27. Morgan DL. Focus groups as qualitative research. 2nd ed. Thousand Oaks, CA. SAGE; 1997.

28. Pfeiffer E. A short portable mental status questionnaire for the assessment of organic brain deficit in elderly patients. J Am Geriatr Soc. 1975;23:433-41.

29. Palinkas LA, Horwitz SM, Green CA, Wisdom JP, Duan N, Hoagwood K. Purposeful sampling for qualitative data collection and analysis in mixed method implementation research. Adm Policy Ment Health. 2015;42:533-44

30. Bajcar J. Task analysis of patients' medication-taking practice and the role of making sense: a grounded theory study. Res Social Adm Pharm. 2006 Mar;2:59-82

31. Shoemaker SJ, Ramalho de Oliveira D, Alves M, Ekstrand M. The medication experience: preliminary evidence of its value for patient education and counseling on chronic medications. Patient Educ Couns. 2011 Jun; 83:443-50.

32. Mohammed MA, Moles RJ, Hilmer SN, Kouladjian O'Donnel L, Chen TF. Development and validation of an instrument for measuring the burden of medicine on functioning and well-being: the MedicationRelated Burden Quality of Life (MRB-QoL) tool. BMJ Open. 2018 Jan 11;8:e018880

33. Fereday J, Muir-Cochrane E. Demonstrating rigor using thematic analysis: a hybrid approach of inductive and deductive coding and theme development. Int J Qual Methods. 2006;5:80-92.

34. Foley G, Timonen V. Using grounded theory method to capture and analyze health care experiences. Health Serv Res. 2015;50:1195-210.

35. Modig S, Kristensson J, Troein M, Brorsson A, Midlov P. Frail elderly patients' experiences of information on medication: a qualitative study. BMC Geriatr. 2012;12:46.

36. Henriques MA, Costa MA, Cabrita J. Adherence and medication management by the elderly. J Clin Nurs. 2012;21:3096-105.

37. Metzl JM, Hansen H. Structural competency: theorizing a new medical engagement with stigma and inequality. Soc Sci Med. 2014 Feb; 103:12633.

38. Spinewine A, Fialova D, Byrne S. The role of the pharmacist in optimizing pharmacotherapy in older people. Drugs Aging. 2012 Jun 1;29:495-510.

39. Verloo H, Chiolero A, Kiszio B, Kampel T, Santschi V. Nurse interventions to improve medication adherence among discharged older adults: a systematic review. Age Ageing 2017;46:747-54.

Publisher's Note Springer Nature remains neutral with regard to jurisdictional claims in published maps and institutional affiliations. 